\title{
FISTULATION PERMANENTE DU PANGRÉAS EXOCRINE CHEZ LE PORC APPLICATION : RÉPONSE DE LA SÉCRÉTION PANCRÉATIQUE AU REPAS
}

\author{
T. CORRING, A. AUMAITRE, et A. RÉRAT \\ avec la collaboration technique de A. M. Gueugneau et P. Vaugelade \\ Station de Recherches sur l'Élevage des Porcs, \\ Centre national de Recherches zootechniques, I. N.R.A., \\ 78 - Jouy-en-Josas
}

\section{RÉSUMÉ}

Dans le but d'étudier, chez le Porc éveillé, la sécrétion pancréatique exocrine et ses va riations en réponse à divers stimuli, il était au préalable nécessaire de mettre au poi nt une technique de fistulation permanente du pancréas chez cet animal.

La technique utilisée est une adaptation de la méthode de McCAUghan (1939). Un cathéter de polyéthylène est introduit dans le canal pancréatique à proximité immédiate de son abouchement intestinal. Un cathéter de polyéthylène de diamètre légère ment supérieur est placé dans le duodénum dans la région voisine de l'abouchement du canal pancréatique et sert à la réintroduction du suc pancréatique. La sécrétion totale de l'organe est recueillie en continu dans u n collecteur de fractions placé dans une enceinte refroidie à $+2^{\circ} \mathrm{C}$. Après échantillonnage pour analyses, le suc est réintroduit en permanence à l'aide d'une pompe.

Dix porcs mâles de race Large White et de poids vif moyen de $40 \mathrm{~kg}$ ont été fistulés sel on cette technique. Les animaux sont isolés de façon à n'être soumis à aucun facteur non contrôlable (proximité d'autres animaux). Ils ont reçu durant l'expérimentation un régime alimentaire renfermant 16 p. Ioo de protéines, distribué en un et deux repas de 30 minutes chacun et à heures fixes dans la journée.

Les résultats ont été obtenus à la suite d'une collecte ininterrompue de suc pancréatique durant 30 à 45 jours.

L'influence du repas se traduit immédiatement après son ingestion par une augmentation de la quantité totale d'enzymes sécrétées. Par ailleurs, le pancréas répond à chaque repas dans la journée.

Cette réponse se manifeste de deux façons différentes :

r. Augmentation du volume sécrété. Activités enzymatiques par $\mathrm{ml}$ de suc pancréatique recueilli constantes.

2. Volume sécrété constant. Augmentation des activités enzymatiques par ml de suc pancréatique recueilli. 


\section{IN'TRODUCTION}

La glande pancréatique exocrine est le siège d'une synthèse protéique très importante et, de ce fait, a toujours suscité l'intérêt des biochimistes. Rappelons que la trypsine est une des premières protéines qui ait été isolée et cristallisée (NoRTHROP et KUNITZ, I932).

Le pancréas exocrine joue un rôle fondamental dans le processus de digestion. En effet, cette glande synthétise et exporte des enzymes agissant sur les trois groupes de substances présentes dans les aliments du monogastrique : lipides, protides et glucides. Ces hydrolases sont véhiculées vers le duodénum dans le suc pancréatique. La teneur en anions de ce dernier lui confère un pouvoir tampon permettant notamment de neutraliser les effets de l'acide chlorhydrique gastrique dans le duodénum. Le suc excrété joue donc un rôle multiple dans la digestion. Cependant 1'aliment peut varier dans sa composition chimique ou par la nature de ses composants et il a été démontré que le pancréas s'adapte à ces variations en modifiant les proportions des enzymes (DESNUELLE et al., I962; HowARD et YUDKIN, I963; SNOOK et MEYER, r964).

Il existe donc une régulation de la sécrétion pancréatique qui permet en particulier cette adaptation et certains facteurs de la régulation ont pu être identifiés et précisés, grâce à l'utilisation de fistules pancréatiques aiguës ou semi-permanentes. Ainsi, Paviov (I893) a mis en évidence l'influence du système nerveux central sur la sécrétion enzymatique. BAyliss et STARLING (I902) avec la sécrétine, HARPER et VASS (I94I) avec la pancréozymine ont montré une influence hormonale respectivement sur les sécrétions hydréolectrolytique et enzymatique du pancréas exocrine. Plus récemment, les travaux de CRIDER et al. (1956) et BEN ABDELJLII, et DESNUELLE (I964) ont introduit le concept de la régulation humorale par les produits de la digestion eux-mêmes.

S'il a été démontré que tous ces facteurs sont mis en jeu soit séparément, soit simultanément au cours de la régulation de la sécrétion pancréatique, on ignore encore aujourd'hui les mécanismes intimes du phénomène.

Les moyens d'exploration sont limités et souvent difficiles à mettre en œuvre. La technique classique du prélèvement du tissu pancréatique ne donne qu'un cliché de la "situation pancréatique " instantanée, après abattage de 1'animal. Une étude de l'évolution de cette situation fait appel à un nombre élevé d'animaux et doit tenir compte des variations individuelles souvent importantes.

Il semble donc nécessaire de pouvoir disposer d'une technique permettant l'étude en continu de la sécrétion pancréatique et de ses variations sous l'influence de stimuli habituels, tels que la prise de nourriture par exemple. Une telle observation pourrait être ainsi répétée dans les mêmes conditions, sur le même animal, et permettrait d'aborder l'étude des mécanismes de la régulation de la sécrétion.

La fistulation permanente du pancréas exocrine paraît être, à l'heure actuelle, la seule technique valable permettant de tester des répétitions du stimulus sur le même animal. Cependant le petit nombre de résultats ob tenus jusqu'à présent prouve qu'elle est difficile à réaliser et à utiliser. 
Thomas (1959) a fait une mise au point exhaustive des différentes méthodes de fistulation permanente du pancréas exocrine et nous en rappellerons l'essentiel. Les méthodes originales décrites par PAVLov (I9IO); INLOW (I92I); DRAGSTEDT et al. (I930); McCaUGHAN (I939), ont permis d'obtenir du suc pancréatique par trois voies essentielles : canulation chronique du canal pancréatique par l'intérieur du duodénum, technique du sac duodénal, fistulation directe du canal pancréatique sans ouverture du duodénum. Par la suite, de nombreuses autres techniques ont été testées mais ne sont que des modifications des précédentes (ANNIS et HALLZNBECK, I95I ; PRESHaw et Grossman, I965; HeRrera et al., I968) sur le chien (MAGEE et Hong, I966 ; PEKas, I965), sur le Porc.

Dans le cadre de nos investigations entreprises sur l'étude de la sécrétion pancréatique chez le Porc, et des facteurs de sa régulation en relation étroite avec 1'alimentation, nous cherchons à disposer d'une technique de fistulation permanente nous permettant d'obtenir du suc pancréatique dans les conditions suivantes :

- la technique utilisée ne doit pas être traumatisante de façon à éviter toute perturbation dans le développement normal de l'animal,

- le Porc doit être éveillé et s'alimenter normalement,

- la sécrétion pancréatique doit être entièrement collectée durant une longue période et le suc obtenu réintroduit au niveau du duodénum.

Après quelques essais, nous avons été amenés à modifier la méthode appliquée au Chien par McCaUghan (I939) et FLoREy et al. (I94I) ainsi que par PEKAS (I965) et Magee et Hong (I966) sur le Porc.

Dans le travail que nous présentons ici, nous nous proposons de décrire cette technique et de montrer l'évolution de la sécrétion pancréatique du Porc, en réponse à l'ingestion d'un repas.

\section{TECHNIQUES ETT SCHÉMA EXPÉRIMENTAL}

\section{Animaux}

Dix porcs mâles castrés de race Large White, de poids vif compris entre 35 et $45 \mathrm{~kg}$, ont été utilisés. Les animaux sont mis en observation et habitués à la contention en cage 8 jours avant l'opération. Durant cette période, ils sont isolés et consomment i à 2 repas par jour, à heure fixe, et pendant une durée limitée à 30 minutes au maximum.

\section{Technique opératoire}

Le principe consiste à dériver totalement la sécrétion pancréatique à l'extérieur de l'animal, afin d'en mesurer le volume sécrété en fonction du temps et de doser la quantité d'enzymes présentes à chaque instant. Après échantillonnage, le suc est réintroduit dans le duodénum.

\section{Préparation des cathéters.}

On utilise du cathéter en polyéthylène (1) de diamètre intérieur et extérieur respectifs de I, 2 et $\mathrm{I}, 7 \mathrm{~mm}$ pour le canal pancréatique et de 1,5 et $2,0 \mathrm{~mm}$ pour la fistule duodénale. Ces cathéters, de longueur moyenne de $50 \mathrm{~cm}$ chacun, sont munis de deux bagues de polyéthylène (schéma I) fixes et situées à l'une des extrémités.

Les cathéters ainsi préparés sont stérilisés, avant emploi, par immersion pendant 24 heures dans une solution de Tétradonium ( ${ }^{(2)}$ à I $\mathrm{p}$. I ooo.

(1) Biotrol, 3, rue de Béarn, Paris.

(2) Laboratoires Avlon, 95 - Enghien. 


\section{Préparation chirurgicale des animaux.}

Avant l'opération, les animaux sont soumis à un jeûne complet de 16 heures. L'anesthésie est réalisée directement et entretenue au masque par un mélange gazeux (oxygène-protoxyde d'azote) barbotant à volonté dans une solution d'Halothane (1).

Le porc est placé en décubitus latéral gauche sur la table d'opération, rasé et désinfecté sur la surface du flanc droit et recouvert de champs opératoires laissant libre accès à cette zone.

\section{Mise en place des cathéters.}

Ier temps opératoire.

La peau et le tissu cellulaire sous-cutané sont incisés sur une longueur de $15 \mathrm{~cm}$ environ parallèlement et légèrement en arrière de la dernière côte flottante. Les différentes couches musculaires sont écartées dans le sens des fibres et le péritoine est ouvert selon l'axe d'incision de la peau.

\section{2e temps opératoire.}

Les lèvres de l'incision sont écartées à l'aide d'un écarteur de Gosset. L'anse duodénale située en partie haute et profonde est saisie et ramenée sans traction vers l'ouverture au moyen de 2 pinces de Bassuet. La partie de l'anse duodénale renfermant la tête du pancréas est ramenée au niveau de l'ouverture. Le canal pancréatique, entièrement enfoui dans le tissu acineux continu jusqu'au duodénum, est mis à jour au niveau de son abouchement intestinal. Il est ensuite dénudé sur une longueur de $0,5 \mathrm{~cm}$ environ (schéma I) en prenant soin de laisser intacte l'irrigation sanguine de l'intestin.

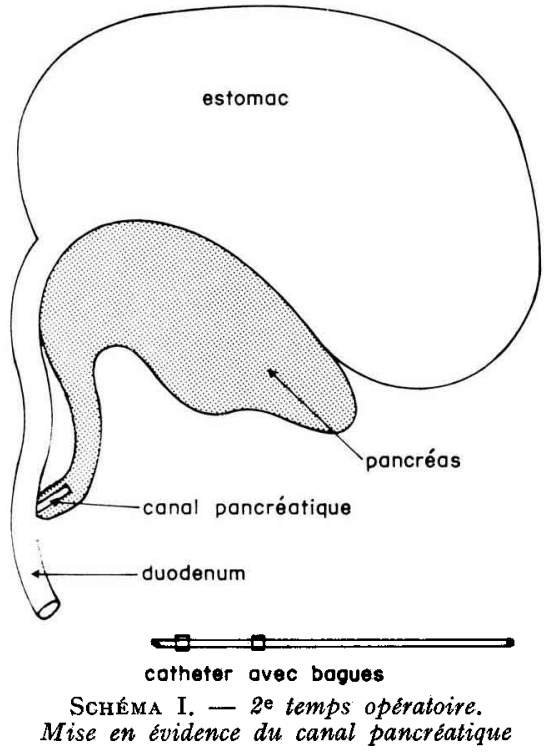

3e temps opératoive.

La partie du canal dénudée est incisée à l'aide de petits ciseaux pointus sur I ou $2 \mathrm{~mm}$ dans le sens transversal. A ce stade généralement quelques gouttes de suc pancréatique apparaissent spontanément au niveau de l'incision, sinon après un léger massage de la tête du pancréas.

Le cathéter pancréatique est introduit, avec précaution, en direction du pancréas et sur une longueur de I à $2 \mathrm{~cm}$ au maximum. La bague la plus proche de l'extrémité est également introduite si le diamètre du canal le permet (ce qui est généralement le cas chez les animaux utilisés). Une ligature en aval de cette bague permet de refermer le canal pancréatique autour du tube de polyéthylène et le cathéter est fixé au duodénum, au niveau de la deuxième bague, par une ligature en bourse auto-serreuse en 8 de chiffre (photo $\mathrm{I}$ ).

Le cathéter duodénal est ensuite introduit dans le duodénum au voisinage de l'abouchement naturel du canal pancréatique. Il est fixé au niveau des deux bagues (la bague proche de l'extrémité est introduite dans l'intestin) par deux ligatures en boucle auto-serreuse en 8 de chiffre (photo 2).

(1) Laboratoires Avlon 95 - Enghien. 
4e temps opératoire.

Un orifice est pratiqué au niveau du flanc droit par ponction au bistouri, permettant d'attirer l'extrémité des 2 canules à l'extérieur de la cavité abdominale (photo 3 ). La partie du duodénum, où sont fixés les 2 cathéters, est ensuite ramenée contre le péritoine, cette opération ayant pour but principal d'éviter qu'une anse intestinale ne vienne ultérieurement s'étrangler autour des tubes de polyéthylène.

\section{$5^{\text {e }}$ temps opératoire.}

La plaie abdominale est refermée par suture des différents plans qui constituent la paroi : suture en surjet (catgut $n^{\circ}$ o) du péritoine, suture par points en $\mathrm{X}$ des aponévroses et des muscles (fil de nylon $n^{\circ}$ 2), suture par points en $U$ de la plaie cutanée (fil de nylon $n^{0} 2$ ).

Les cathéters sont fixés à la peau à l'aide d'un nœud auto-serreur.

Soins post-operratoires.

Les infections péritonéales sont prévenues à 1'aide de trois injections intramusculaires de pénicilline-retard (I million UI) et de streptomycine (I g) à 24 heures d'intervalle après l'opération. En outre, I million UI de pénicilline en poudre sont placées à l'intérieur de la cavité abdominale, avant la suture du péritoine.

Durant toute l'expérimentation, les infections microbiennes éventuelles sont surveillées par la prise régulière de température.

\section{Fistule pancréatique.}

Utilisation des fistules

Le suc pancréatique est recueilli en continu dans un collecteur de fractions ( ${ }^{1}$ ) maintenu à $+2^{\circ} \mathrm{C}$. Le collecteur comprend $\mathrm{r} 80$ tubes de $35 \mathrm{ml}$ gradués en $0,5 \mathrm{ml}$ et la durée de collecte, réglable, est en général, pour les animaux utilisés, de ro à 15 minutes par tube.

\section{Fistule duodénale.}

Après les mesures volumétriques et l'échantillonnage pour les déterminations chimiques et enzymatiques, le suc pancréatique, ramené à la température normale $\left(3^{\circ} \mathrm{C}\right)$, est réintroduit à l'aide d'une pompe aspirante et refoulante $\left({ }^{2}\right)$ à débit variable (0-1 $50 \mathrm{ml} / \mathrm{heure}$ ) par la canule duodénale. La réintroduction est effectuée à débit constant durant les 6 heures qui suivent le repas et la quantité de suc réintroduit est égale à celle recueillie la veille pendant la même période.

\section{Schéma expérimental}

Les animaux opérés ne sont mis en expérience que le $6^{\mathrm{e}}$ jour suivant l'opération. Auparavant, les deux cathéters sont directement reliés afin d'assurer le retour immédiat du suc pancréatique à l'animal.

Nous n'avons jamais noté de reflux duodéno-pancréatique, évitant ainsi l'utilisation d'une valve sans reflux (PEKAs (1965)).

\section{TABLEAU I}

Composition en p. 100 de la matière fraîche de l'aliment expérimental

\begin{tabular}{|c|c|}
\hline Matière sèche $\ldots \ldots \ldots \ldots \ldots \ldots \ldots \ldots \ldots$ & 88,04 \\
\hline Matières azotées $(\mathrm{N} \times 6,25) \ldots \ldots \ldots \ldots \ldots \ldots$ & 16,86 \\
\hline Matières grasses $\ldots \ldots \ldots \ldots \ldots \ldots \ldots \ldots \ldots$ & 2,11 \\
\hline 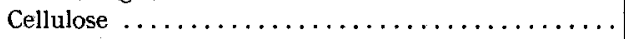 & 4,85 \\
\hline 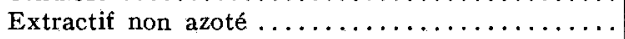 & 57,94 \\
\hline 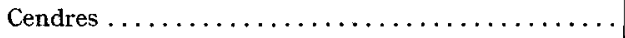 & 6,28 \\
\hline
\end{tabular}

(1) Collecteur Seive, Paris.

(2) Micropompe D. C. L. Comedo, 9 I - Corbeil. 

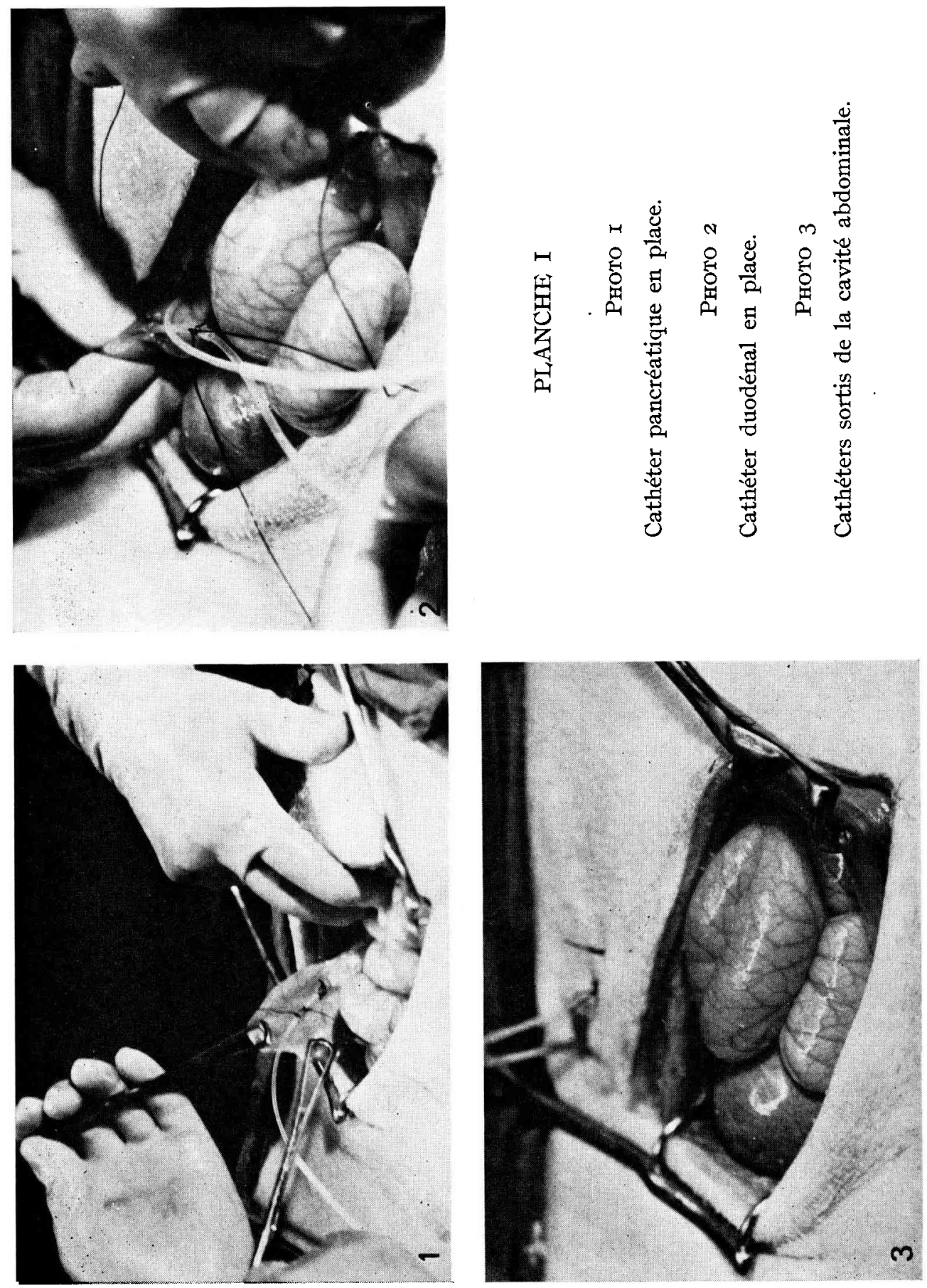
Sur les Io porcs utilisés, 7 ont reçu un repas de $900 \mathrm{~g}$ par jour et 3 ont reçu deux repas de $450 \mathrm{~g}$ chacun, par jour, d'un aliment équilibré (tabl. I), présenté sous forme de farine mélangée à l'eau $(\mathrm{p} / \mathrm{p} \mathrm{I}-\mathrm{r})$.

Les animaux sont isolés de façon à ne pas être soumis à des stimuli tels que le rythme d'alimentation d'autres animaux, la présence de l'homme, etc. De façon générale, le repas fait suite ou précède une longue période dè sommeil, représentant le comportement normal du porc dans les conditions habituelles.

\section{Déterminations volumétrique et enzymatiques}

Le volume du suc pancréatique recueilli est exprimé en $\mathrm{ml}$ de suc par heure. Les analyses chimiques sont effectuées sur toutes les fractions cumulées. Sur chacun de ces échantillons ont été déterminées la teneur en protéines et les activités enzymatiques de la chym ətrypsine, de la trypsine et de l'amylase. Le principe du dosage des enzymes protéolytiques est donné dans un travail précédent (CoRRING et AUMAITRE, 1970). L'activation du chymotrypsinogène et du trypsinogène du suc est effectuée à l'aide de la trypsine (1), à raison de $5 \mathrm{mg}$ et $10 \mathrm{mg}$ respectivement pour roo mg de protéines du suc pancréatique. Le dosage de l'activité amylasique est effectué selon la méthode de MetraIs sur le sérum (I966) que nous avons modifiée (CoRRING et SaUtCIER, non publié) en raison des fortes activités rencontrées dans le suc pancréatique. En particulier toutes les dilutions se font dans $\mathrm{NaCl} 0,9 \mathrm{p}$. Ioo et la quantité de substrat a été augmentée ainsi que la concentration de l'acide chlorhydrique (I N contre $0, \mathbf{I} N$ ). Le substrat est l'amidon soluble MercK (no 1252), préparé à I p. roo dans un tampon phosphate $\mathrm{pH}$ 7,15. L'emploi d'autres amidons a été moins satisfaisant. La solution d'amidon préparée se conserve parfaitement à la température ambiante durant 3 semaines au moins. L'unité déterminée selon cette méthode représente l'unité d'activité amylasique contenue dans $\mathrm{r}, 0 \mathrm{ml}$ de suc pancréatique et qui hydrolyse I,o $\mathrm{mg}$ d'amidon soluble Merck, durant 30 minutes à $37^{\circ} \mathrm{C}$.

Les activités enzymatiques sont exprimées à la fois en activités totales (activités par $\mathrm{ml}$ de suc $\times$ volume de l'échantillon) et en activités spécifiques (activités/mg protéines).

\section{RÉSULTATS}

La croissance des animaux durant l'expérimentation n'a apparemment pas été perturbée par l'intervention chirurgicale, puisque nous avons enregistré des gains quotidiens de l'ordre de 300 à $500 \mathrm{~g}$.

La durée moyenne d'utilisation des canules a été de 35 jours, période après laquelle nous avons constaté un rejet progressif du cathéter pancréatique pour la plupart de nos animaux. Ce rejet s'accompagne en général de la mort des sujets ; 1'autopsie révèle alors une atrophie presque complète du tissu pancréatique ainsi qu'une augmentation très importante du volume du canal pancréatique. Très souvent également nous avons observé un début de digestion tissulaire au voisinage immédiat de l'implantation de la canule pancréatique.

Ainsi, les valeurs des mesures effectuées peuvent être groupées en deux types de résultats :

atypiques,

typiques.

Le premier type se retrouve parmi les données obtenues durant les jours qui suivent l'opération, ceux qui précèdent le rejet de la canule ou encore lorsque l'animal perd du poids ou montre une croissance anormale. La sécrétion pancréatique présente alors des variations inexplicables d'un jour à l'autre et même au cours de la

(1) Trypsine Worthington. 
journée. Il semble bien que le mauvais état physiologique de l'animal (conséquences post-opératoires, croissance perturbée) induise un disfonctionnement de la sécrétion pancréatique. De plus, pour l'ensemble de nos animaux, la quantité totale de protéines exportée augmente régulièrement dans les 5 jours qui suivent l'opération. Elle atteint une valeur stable qui se maintient par la suite, et l'on peut supposer que l'animal a retrouvé son état physiologique normal 5 jours après la fistulation.

Les données du groupe dit typique ont été obtenues sur une période de 20 jours en moyenne pour un ensemble de résultats cohérents, parfaitement répétables dans le temps.

Nous avons pu ainsi, et malgré les variations individuelles importantes, observer une évolution comparable de certains phénomènes en fonction du repas, indépendamment de 1'animal.

\section{A. - Évolution de la sécrétion enzymatique du pancréas en réponse au repas}

Nous avons reporté l'évolution de la quantité de protéines du suc pancréatique recueilli par heure au cours de la journée (fig. I et 2 ).

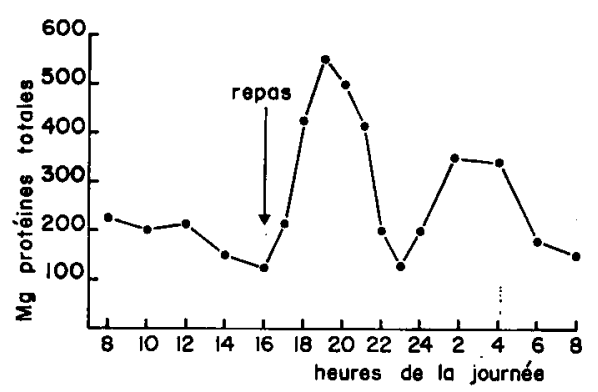

FIG. I. - Infuence du repas sur l'éolution de la quantité de proteines totales du suc pancréatique chez le Porc (I seul repas/jour)

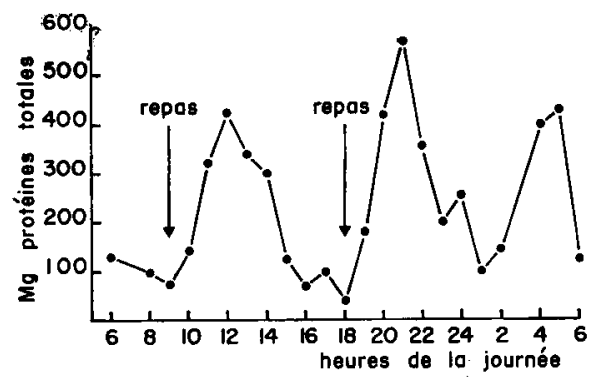

FIG. 2. - Influence de la fréquence de distribution des repas sur l'évolution de la quantité de protéines totales du suc pancreatique chez le Porc (2 repas/jour)

Nous constatons que la réponse du pancréas à la prise de nourriture est immédiate et se manifeste par une augmentation importante de la quantité de protéines exportée. Cette augmentation se poursuit durant 5 à 7 heures après le début du repas et présente un maximum à la $3^{\text {e heure. }}$ 

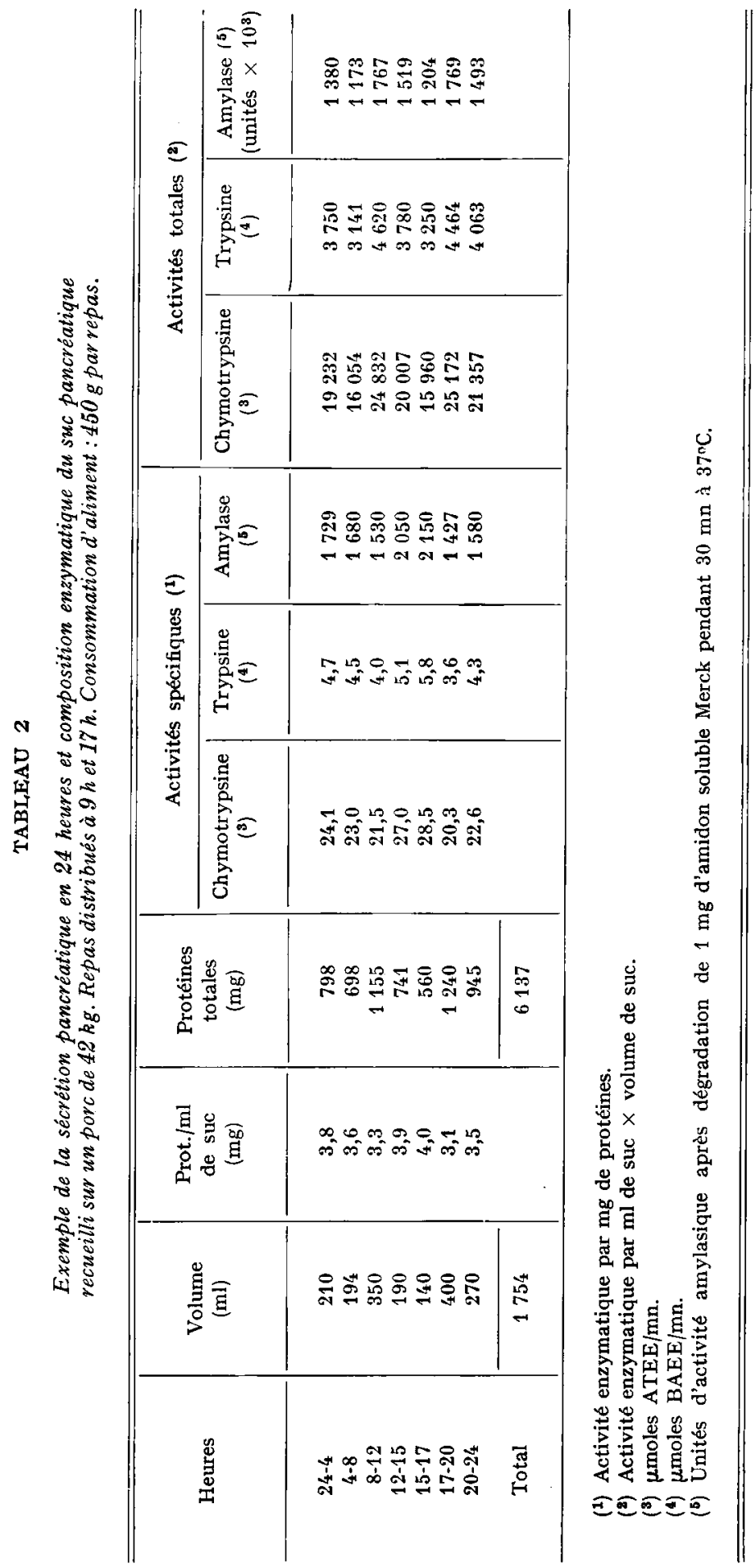
Dans certains cas, nous avons même noté une élévation de la sécrétion enzymatique dans les 5 minutes qui ont précédé le repas. Quel que soit le nombre de repas distribués, le pancréas répond à chacun d'entre eux (fig 2). On peut souligner que la quantité de protéines excrétées en 24 heures est indépendante de la fréquence de distribution des repas ; elle est en moyenne de 5 à $7 \mathrm{~g}$ (tabl. 2) pour une même quantité totale d'aliment ingéré par jour, consommée en un ou deux repas.

Enfin, et quelle que soit 1'heure de distribution du repas, nous avons noté, sur tous les animaux, une augmentation de la sécrétion enzymatique au milieu de la nuit. Les valeurs enregistrées peuvent être identiques à celles obtenues les trois premières heures après le repas.

\section{B. - Modalités de la réponse pancréatique au repas}

Nous avons observé deux types de réponse consécutive à l'ingestion du repas et cela chez des animaux différents.

Io Dans le $\mathrm{I}^{\mathrm{er}}$ type, le repas stimule fortement la sécrétion aqueuse du pancréas tandis que la concentration en protéines du suc demeure constante. Ainsi (fig. 3), le volume recueilli est de l'ordre de 30 à $40 \mathrm{ml} /$ heure avant le repas et atteint $5_{50}$ et même $200 \mathrm{~m} 1 /$ heure dans les 3 heures qui suivent la prise de nourriture.

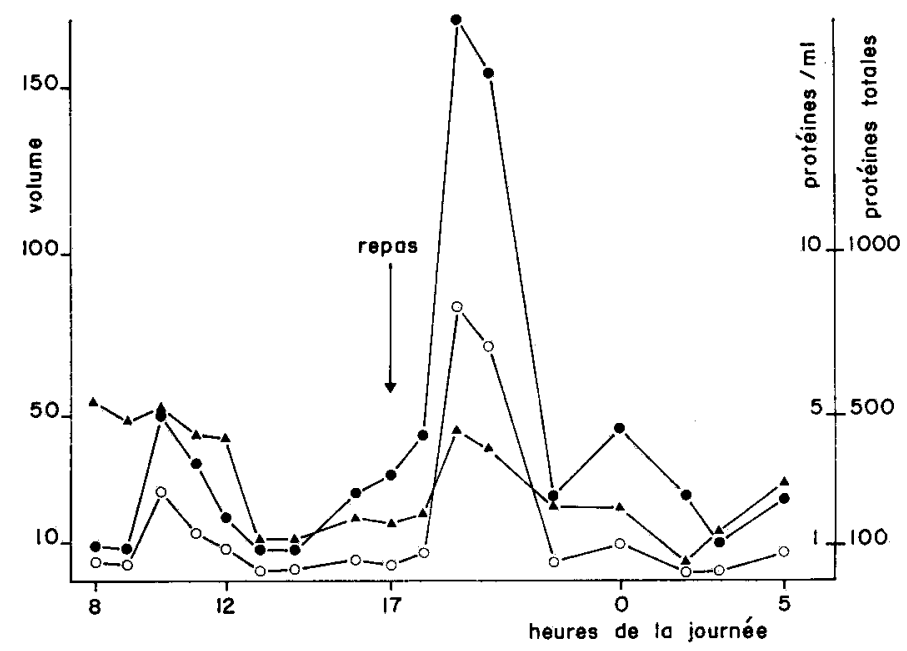

FIG. 3. - Influence du repas sur la sécrétion pancréatique chez le Porc (I er type de réponse)

mg de Protéines par $\mathrm{ml}$
volume en ml
Protéines totales en $\mathrm{mg}$

$2^{\circ}$ Dans un $2^{e}$ type de réponse (fig. 4), nous assistons au phénomène exactement inverse : à la suite du repas, le volume sécrété demeure constant ou diminue légèrement. Mais on note une augmentation de la teneur en protéines du suc qui peut atteindre Io à $I 5$ fois les valeurs moyennes obtenues en dehors du repas.

Ainsi, quel que soit le mode de réponse, la quantité de protéines enzymatiques exportées augmente fortement sous 1 'influence du repas. 


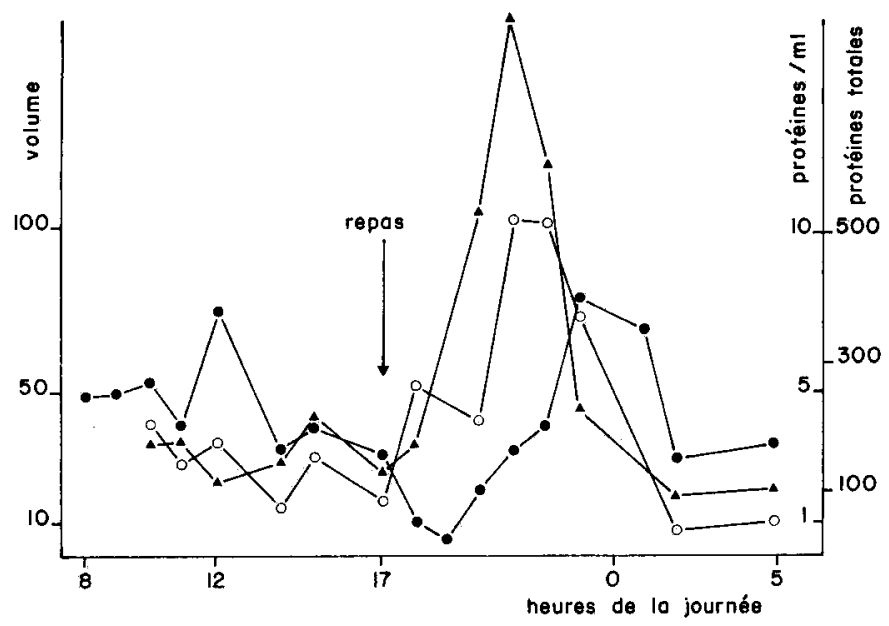

FIG. 4. - Infuence du repas sur la sécrétion pancréatique chez le Porc ( ${ }^{\mathrm{e}}$ type de réponse)

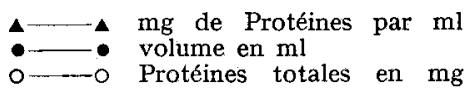

Un exemple chiffré de ces principaux phénomènes (réponses volumétrique et enzymatique) est donné dans le tableau 2 qui rassemble les principales valeurs enregistrées au cours d'une journée sur l'un de nos animaux. La réponse aux repas est du I $^{\text {er }}$ type décrit et concerne une augmentation du débit du suc associé à une teneur en protéines du suc constante, sinon en diminution. Ainsi les volumes enregistrés passent de $195 \mathrm{ml}$ à $350 \mathrm{ml}$ au terme du premier repas et de $\mathrm{I} 40 \mathrm{ml}$ à $400 \mathrm{ml}$ lors du second repas. La concentration du suc en protéines pour ces mêmes périodes passe de $3900 \gamma / \mathrm{ml}$ à $2750 \mathrm{\gamma} / \mathrm{ml}$ et de $4400 \mathrm{\gamma} / \mathrm{ml}$ à $2300 \mathrm{\gamma} / \mathrm{ml}$. Outre les informations concernant le niveau des activités spécifiques et totales, il est remarquable de noter le parallélisme dans les variations de la teneur en protéines et des activités enzy matiques totales ou spécifiques du suc.

\section{DISCUSSION}

\section{Technique opératoire}

Les principales observations réalisées nous permettent de mieux définir les conditions de mesure de la sécrétion pancréatique chez le Porc. L'animal doit être en bonne santé, s'alimenter normalement, et la sécrétion pancréatique doit pouvoir être recueillie en continu pendant une longue période, nécessitant une réintroduction du stuc dans le duodénum, pour éviter de perturber la digestion. Compte tenu de ces conditions, les méthodes initiales de fistulation décrites et les modifications apportées par la suite ne peuvent être appliquées. Ainsi, la technique de fistulation du canal pancréatique par l'intérieur du duodénum ('THOMAs, I959) entraîne une augmenta- 
tion du traumatisme opératoire et un risque de formation de volvulus intestinal et d'occlusions. De plus, la fixation dans le méat pancréatique peut être insuffisante età l'origine de collectes non quantitatives de la sécrétion, ce que l'on peut déduire des résultats obtenus par deux d'entre nous (AUMAITRE et RÉRAT, I969).

Par ailleurs, quel que soit son mode d'introduction dans le canal pancréatique (incision du duodénum ou cannulation directe), le cathéter peut être la cause d'une stimulation de la sécrétion par voie nerveuse et d'une hypersécrétion. C'est en effet ce que nous avons observé durant les 5 jours qui suivent 1'opération. Mais il s'agit principalement d'une hypersécrétion de la partie liquide du suc, qui disparaît par la suite, comme l'indiquent la cohérence et la stabilité de nos résultats.

La technique du sac duodénal peut pallier cet inconvénient (PRESHAW et GrossMAN, r965). Cependant, on ignore absolument l'effet de cette méthode sur la synthèse et la sécrétion des hormones intestinales (sécrétine, pancréozymine), ainsi que les répercussions d'une section du système nerveux irrigant l'intestin, sur la sécrétion pancréatique elle-même.

Les résultats que nous avons obtenus jusqu'à présent se rapportent plus précisément à des animaux réalisant un gain de poids considéré comme normal et de 1'ordre de Io $\mathrm{kg}$ en un mois. Par ailleurs, les porcs se sont réalimentés normalement dès le $2^{\mathbf{e}}$ jour après 1'opération. Cependant, la question peut être la plus importante est l'état du pancréas en fin d'expérimentation; la glande pancréatique peut parfois disparaître alors que le canal pancréatique a subi une hypertrophie considérable. Cette observation avait déjà été faite chez le Chien par TrortskaIa et FunTrKova (I965), mais nous ne pouvons donner aucune explication à ce phénomène.

Compte tenu de ces diverses observations, on peut considérer que les résultats exploitables portent sur une période moyenne de 20 jours, dans les conditions actuelles d'utilisation de la technique de fistulation. Nous avons, en effet, observé durant cette période une répétabilité des valeurs (volume de suc recueilli ou teneur en protéines totales) pour un même animal, et cela sur tous les animaux.

Il importe d'insister sur un autre point qui concerne la récolte du suc pancréatique et sa réintroduction. On ne peut, en effet, être certain de recueillir la totalité de la sécrétion pancréatique en raison de la présence possible de un et même deux canaux pancréatiques accessoires chez le Porc (Vodovar et al., I964).

En ce qui concerne le problème de la nécessité d'une réintroduction du suc; il semble bien établi à présent (Thomas, I959) que chez quelques espèces, un animal privé de sa sécrétion pancréatique meurt rapidement. Il s'agirait beaucoup plus d'une mort par perte d'éléments hydro-électrolytiques que par insuffisance enzymatique. Par exemple, un porc de $40 \mathrm{~kg}$ peut exporter dans le suc pancréatique 7 à Io $\mathrm{g}$ d'ions sodium (CORRING et GUEGUEN, I97I). Néanmoins, rien ne nous autorise à sous-estimer le rôle du suc dans la digestion. Quelques observations nous démontrent, en effet, que l'absence de réintroduction prolongée pendant 2 jours est très souvent suivie d'une diarrhée très importante, qui cesse dès la première réintroduction de suc. Les techniques de fistulation faisant intervenir une canule à 2 voies (Thomas, I959) ou une valve à sens unique (PEKAS, I965), permettent d'éviter la réintroduction mais interdisent la mesure quantitative de la sécrétion.

Nous pouvons conclure que la technique que nous avons utilisée nous a permis d'atteindre les deux buts essentiels : disposer d'un animal dans les conditions physiologiques normales et recueillir le suc pancréatique en continu. 


\section{Influence de la prise de nourriture sur la sécrétion pancréatique}

De l'ensemble des données obtenues se dégagent nettement deux effets principaux de la prise de nourriture sur l'évolution de la sécrétion pancréatique chez le Porc.

En premier lieu, nous avons montré l'augmentation immédiate de la quantité d'enzymes exportées dès le début du repas. HERRERA et al. (Ig68) sur le Chien, PEKAS (I96I) sur le Porcelet ont obtenu des résultats comparables, mais n'ont pas mis en évidence la régularité de la durée de cette réponse. Nous avons noté qu'elle se poursuivait durant 5 à 7 heures après être passée par un maximum à la $3^{\mathrm{e}}$ heure, phénomène entrevu par PEKAS malgré de très importantes variations.

Ce type de résultat souligne le rôle du pancréas dans les phénomènes de la digestion. En effet, on constate un parallélisme étroit entre l'augmentation de la sécrétion et l'évacuation gastrique alimentaire chez le Porc, maximum durant les trois premières heures suivant le repas et qui se poursuit à un rythme plus faible jusque vers la

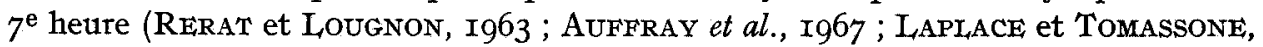
I $970 \mathrm{a}$ ). Ce phénomène n'a, par contre, pas été mis en évidence chez le porcelet plus jeune par PEKAS (I96I), soit en raison des grandes variations existant entre les mesures, soit à cause de l'accélération de l'évacuation gastrique observée par KIDDER et MANNERs (Ig68).

En ce qui concerne la réponse globale du pancréas à la prise de nourriture, quelques observations nous permettent de noter une augmentation de la quantité de protéines exportée dans les 5 minutes qui précèdent le début du repas. La présence de l'homme, la préparation du repas peuvent être à l'origine de ce phénomène et apportent un argument en faveur de l'existence d'une régulation nerveuse de la sécrétion pancréatique avancée par PRESHAw et al. (I966) et PEKAS (I96I). Cette augmentation pourrait encore être expliquée par l'évacuation du "fonds " de l'estomac observée en général avant le repas de l'animal (AUFFray et al., I967).

Par ailleurs, nous n'observons pas de variations de la sécrétion pancréatique (quantité de protéines excrétées) avec le nombre de repas, lorsque la quantité d'aliment consommé est identique. Ce résultat est à rapprocher de celui de MAGEE et HoNG (1959) montrant une augmentation significative de la sécrétion pancréatique (volume et concentration enzymatique) chez le Chien lorsque la quantité d'aliment ingéré passe de 450 à $900 \mathrm{~g}$ par jour.

Enfin, nous avons noté l'apparition fréquente d'un pic de sécrétion important au milieu de la nuit, et quelle que soit 1'heure de distribution du repas dans la journée. Nous ne pouvons donner d'explication à ce phénomène mais simplement signaler la mise en évidence d'une importante motricité intestinale (LAPLACE, I970 $b$ ) et de variations importantes du débit du sang porte (RÉRAT, I97I) durant cette même période. Dans le cas d'une motricité intestinale accrue, l'idée d'une stimulation nerveuse de la sécrétion peut encore être considérée. Dans le cas du débit sanguin, la relation, par contre, est plus difficile à établir. En effet, les nombreux travaux effectués dans ce but (JACOBSON, I967) conduisent à des résultats extrêmement contradictoires.

Le deuxième effet de la prise de nourriture sur la sécrétion est le mode de réponse du pancréas. A notre connaissance, aucun travail n'a montré la dualité de cette réponse que nous avons mise en évidence ici. Le pancréas exporte une quantité impor- 
tante d'enzymes après la prise de nourriture en augmentant soit le volume sécrété, soit la concentration en enzymes du suc. Seul PEKas (I96I) a observé, malgré l'hétérogénéité des résultats, une certaine évolution des concentrations enzymatiques dans le suc pancréatique recueilli. Cependant, les porcelets ont été utilisés trop tôt après l'opération et les résultats ne présentent pas un caractère de répétabilité et de stabilité, s'apparentant ainsi aux données que nous avons décrites comme atypiques.

Les modalités de la réponse pancréatique, telle que nous l'avons décrite, montrent que la sécrétion pancréatique est soumise à une régulation qui fait penser à l'action hormonale de la sécrétine (variation du volume) ou de la pancréozymine (variation de la teneur en enzymes). Malheureusement, ce travail ne nous permet pas de préciser et d'identifier les facteurs en cause ni d'expliquer les mécanismes de cette régulation particulière de la sécrétion pancréatique en réponse à la prise de nourriture.

Enfin, il est remarquable de constater (tabl. 2) que la teneur en protéines et les activités enzymatiques par $\mathrm{ml}$ de suc varient toujours dans le même sens, confirmant les résultats de KELIER et al. (1958).

\section{CONCLUSION}

Une technique de fistulation du pancréas exocrine du Porc a permis de recueillir en continu, et sur une longue période, du suc pancréatique sur un même animal, qui s'alimente normalement et dont la croissance est normale.

Les résultats obtenus ont mis en évidence quelques phénomènes stables et répétables dans la réponse du pancréas à la prise de nourriture. Ainsi, avons-nous montré que le pancréas sécrète une grande quantité d'enzymes dès le début du repas et sur une période de 5 à 7 heures. L'augmentation de l'exportation d'enzymes s'explique par une variation du volume de suc à concentration constante en protéines, ou par une augmentation de la teneur en protéines par millilitre de suc, à volume constant.

Cette technique a permis de réaliser quelques observations préliminaires avant d'aborder, par la suite, l'étude de la régulation de la sécrétion pancréatique, notamment dans le cas de l'adaptation du régime alimentaire.

$$
\text { Reçu pour publication en aonit } 1971 .
$$

\section{SUMMARY}

\section{PERMANENT PANCREATIC FISTULATION IN THE PIG SECRETORY RESPONSE TO MEAL, INGESTION}

A technique for permanent pancreatic fistulation was investigated in view of studying pancreatic exocrine secretion in the unanaesthetized Pig.

Our technique is a modified Mc CAUGHaN's technique (1939) using a polythene catheter inserted into the pancreatic duct near its duodenal opening, and another slightly bigger catheter inserted in the vicinity of the pancreatic duct opening to allow pancreatic juice to flow into the intestine. Total pancreatic secretion was permanently collected in a $2^{\circ} \mathrm{C}$-cooled sample collector ; after sampling for analysis, pancreatic juice was permanently flown into the intestine by mean of a pump. 
Io Large White pigs of $40 \mathrm{~kg}$ average live weight were fistulated and were isolated so that no environmental factor like presence of other animals could interfere with the experiment. They were fed with a 16 per cent protein diet given in one or two $30 \mathrm{mn}$ meals at scheduled times of day.

Our results were obtained from 30 to 45 days long permanent collections.

Food ingestion presently induced an increase in total secretory enzymes after each meal in a day. This increase was due either to an increase in the amount of secretion with constant enzyme activity per ml of juice, or to an increase in enzyme activity per ml of juice with constant amount of secretion.

\section{RÉFÉRENCES BIBLIOGRAPHIQUES}

Annis D.; Hallenbeck G. A., 195I. Effect of excluding pancreatic juice from duodenum on secretory response of pancreas to a meal. Proc. Soc. Exptl. Biol., Med., 77, 383-385.

Auffray P., Martinet J., Rerat A., I967. Quelques aspects du transit gastro-intestinal chez le Porc. Ann. Biol. anim. Bioch. Biophys., 7, 261-279.

Aumaitre A., Rerat A., I969. Le développement des enzymes dans le tube digestif du jeune porcelet : importance pour le sevrage et signification nutritionnelle. In Congrès F. E. Z. Commission Production Porcine, Helsinki.

Bayliss W. M., Starling E. H., rgoz. The mechanism of pancreatic secretion. J. Physiol, London, 28, 325-353.

Ben Abdeljlil A., Desnuelle P., 1964. Sur l'adaptation des enzymes exocrines du pancréas à la composition du régime. Biochim. Biophys., Acta, 81, 136-149.

Corring T., Aumaitre A., r97o. Mise en place et évolution de l'équipement enzymatique du pancréas exocrine du jeune Rat pendant la période embryonnaire, l'allaitement et le sevrage. Ann. Biol. anim. Bioch. Biophys, 10, 43 I-44I.

Corring T., Gueguen L., Ig7i. Données non publiées.

Crider J. O., Conly S. S., Dorchester J. E., Thomas J. E., 1956. Effect of I. V. injection of hypertonic glucose solution on external secretion of pancreas. Amer. J. Physiol., 186, 187-189.

Desnuelle P., Reboud J. P., Ben A bDeljlil. A., 1962. Influence of the composition of the diet on the enzyme content of rat pancreas. In : Ciba Foundation Symposium on the exocrine pancreas, Churchill (London), p. 90-107.

Dragstedt L. R., Montgomery M. L., Ellis J. C., 1930. A new type of pancreatic fistula. Proc. Soc. Exptl. Biol. Med., 28, I09-г го.

Florey H. W., Wright R. D., Jennings M. A., I941. Secretions of the intestines. Physiol. Rev., 21, 36-39.

Harper A. A., VAss C. C. N., I94I. The control of the external secretion of the pancreas in rats. J. Physiol, Lonäon, 99, 415-435.

Herrera F., Kemp D. R., Tsukamoto M., Woonward E. R., Dragstedt L. R., I968. A new canula for the study of pancreatic function. J. Appl. Physiol., 25, 207-209.

HowARD F., YUDKIN J., 1963. Effect of dietary change upon the amylase and trypsin activities of the rat pancreas. Brit. J.Nutr., 17, $28 \mathrm{I}-294$.

Inlow W., de P., I92I. Technic for establishment of a permanent pancreatic fistula with secretion of inactive proteolytic ferment. J. Lab. Clin. Med., 7, 86 .

JACOBson E. D., I967. Secretion and blood flow in the gastrointestinal tract. In : Handbook of Physiology section 6 ; Alimentary Canal, Vol. 11, I043-ro62.

Keller P. J., Cohen E., Neurath H., I958. The proteins of bovine pancreatic juice. J. Biol. Chem., 233, 344-349.

Kidder D. E., Manners M. J., 1968. Passage of food in the young suckling pig. Proc. Nutr, Soc., 27, $46 \mathrm{~A}$.

Laplace J. P., Tomassonne R., I970a. Évacuation gastroduodénale chez le Porc : fistulation chronique par voie thoracique extra pleurale; recherches d'une technique d'analyse mathématique de l'évacuation. Ann. Zoot., 19, 303-332.

LAPdace J. P., I970 $b$. Communication personnelle.

Mc CAUGhan J. M., I939. Experimental pancreatic fistula ; simple and satisfactory method for investigating external function of the pancreas. Am. J. Digest. Dis., 6, 392.

MageE D. F., Hong S. S., I959. Daily output of pancreatic juice and some dietary factors which influence it. Am. J. Physiol., 197, 27-30.

MAGeE D. F., HoNG S. S., I 966 . Studies on pancreatic physiology in pigs. In : Swine in biomedical research, Ed. Bustad L. K., Mc Clellan R. O., pp. 109-118.

Metais P., Bieth J., ig68. Détermination de l'a-amylase par une micro-technique. Ann. Biol. Clin., 26, I33-I 42 . 
Northrop J. H., Künitz M., 1932. Crystalline trypsin. Isolation and tests of purity. J. Gen. Physiol., 16, 267-295.

Pavlov I. P., x893. Beiträge zur Physiologie der absonderungen Innervation der Bauchspeicheldrüse. Arch. Physiol., Suppl., 176-200.

Pavlov I. P., Igio. The work of the digestive glands (2nd ed.), translated by W. H. Thompson. Philadelphia Lippincott, 59-64.

Pekas J. C., r96r. Development of exocrine secretion of the pancreas and nutrient utilization in the postnatal pig. Ph. D. thesis. Iowa State Univ. Sci. Ames.

Pekas J. C., 1965. Permanent pancreatic fistula of the pancreas and other digestive glands. J. Appl. Physiol., 20, 1082-1084.

Preshaw R. M., Grossman M. I., 1965. Stimulation of pancreatic secretion by extracts of the pyloric gland area of the stomach. Gastroenterology, 48, 36-44.

Preshaw R. M., Cooke A. R., Grossman M. I., 1966. Sham feeding and pancreatic secretion in the dog. Gastroenterology, 50, 171-178.

Rerat A., Lougnon J., I963. Études sur le transit digestif chez le Porc. Ann. Biol. anim. Bioch. Biophys., 8 (no h. s. i.), 2 I-3o.

Rerat A., r97r. Mesure du débit de sang dans la veine porte à l'aide d'un débitmètre électromagnétique chez le Porc. Ann. Biol. anim. Biochim. Biophys., 11, 175-180.

Snook J. T., Meyer J. H., I964. Responses of digestives enzymes to dietary protein. J, Nutr., 82, 409-4I3.

Thomas J. E., I959. Methods of collecting pancreatic juice. Gastroenterology, 36, 362-367.

Troitskaia V.B., Funtikova E. K., 1965. Pancreatic secretion during development of atrophý caused by major duct fistula. Fiziol. Zh. S.S.S. R. Sechenov., 51, 1327-34.

Vodovar N., Flanzy J., Françors A. C., I964. Intestin grêle du porc. I. Dimensions en fonction de l'âge et du poids ; étude de la jonction du canal cholédoque et du canal pancréatique à celui-ci. $A n n$. Biol. anim. Bioch. Biophys., 4, 27-34. 